\title{
Impact of Bar-Code Medication Administration and Electronic Medication Administration Record System in Clinical Practice for an Effective Medication Administration Process
}

\author{
Mohanaa Naidu, Yeo Lee Yean Alicia \\ Health Service Management, Griffith University, Queensland, Australia \\ Email: mona7178@gmail.com
}

How to cite this paper: Naidu, M. and Alicia, Y.L.Y. (2019) Impact of Bar-Code Medication Administration and Electronic Medication Administration Record System in Clinical Practice for an Effective Medication Administration Process. Health, 11, 511-526.

https://doi.org/10.4236/health.2019.115044

Received: March 29, 2019

Accepted: May 18, 2019

Published: May 21, 2019

Copyright $\odot 2019$ by author(s) and Scientific Research Publishing Inc. This work is licensed under the Creative Commons Attribution International License (CC BY 4.0).

http://creativecommons.org/licenses/by/4.0/

\section{Open Access}

\begin{abstract}
Clinical innovations are ideations resulting from collective experiences that enhance the "norm" and embrace an avenue for change with an invention. As such, collective data that were suggestive of increased medication errors that compromised patient safety initiated the exploration of methods that could reduce multifactorial human errors. The pursuit for an appropriate system followed with the discovery of barcode medication administration system (BCMA) and electronic medication administration system (e-MAR). Prior to the adoption of BCMA and e-MAR, it was crucial to assess the impact of the new medication administration system and the rate of medication administration errors recorded, specifically those that resulted in harm. The purpose of the study was to evaluate BCMA and e-MAR usage outcomes, clinical practices, policies, and processes impacting nurses administering medications in the clinical environment using BCMA and e-MAR system. Thus, an annotated literature review was conducted prior to the implementation of the innovation which analyzed various studies that elaborated on their study methods, data collection and analysis that deliberated on the advantages and disadvantages of barcode medication administration system. It is evident in the researched journals that increased compliance was observed with appropriate guidance, processes and policies in place. There was also a significant reduction in reported errors. The incorporation of barcode technology with electronic medication administration record (e-MAR) had greatly improved the efficiency of the BCMA system. BCMAs method was one of the proposed solutions to medication administration errors and to enhance patient safety measures. As such, the innovation could significantly reduce medication error resulting from intrinsic and extrinsic factors. This paper will further ela-
\end{abstract}


borate on the advantages and disadvantages of BCMA and e-MAR, the strategies assumed in the development of BCMA and e-MAR system and its implementation process by identifying and overcoming potential challenges that may arise.

\section{Keywords}

Electronic Medication Record, Barcode Medication Administration, Medication Errors

\section{Introduction}

Medication use is a complex process involving medical practitioners, pharmacists and nurses. It is an undeniable fact that medication errors occur in all health care settings. Several steps in the entire process such as transcription, dispensing and administration process may have potential risks for serious errors and patient harm [1] [2]. These errors may occur due to human factors that are caused by fatigue, workload, negligence, overlooked information, lack of knowledge and concentration while handling medications [3] [4]. Many studies were conducted, and findings have revealed that large numbers of hospitalized patients are harmed, injured or have experienced adverse drug event (ADE) because of medication errors [5] [6]. Hence, it is crucial to identify and address the risks as it may aid in the prevention of such occurrences. As such, data collected from hospital occurrence reports submitted by an anonymous organization revealed that there were (9) 35\% near misses and (17) 65\% medication errors reported between January to December 2016. The findings proved the need for an improved medication management system. Consequently, Barcode Medication Administration (BCMA) and electronic Medication Administration Record (e-MAR) technologies were identified as two ways to improve inpatient medication safety by automating the process of medication verification and using electronic medication administration record.

The aim of the clinical innovation is to assist nurses in the process of medication administration through the use of the BCMA and e-MAR system to prevent unduly medication errors/near-misses and develop a patient safety culture in the hospital. The goal of the BCMA and e-MAR system is to ensure that the right patient receives the correct medications adhering to the five rights with an electronic validation and documentation system. The information encrypted in barcodes on each medication pack and the patient's barcode identification tag allow the nurses to scan and verify the required details before each administration. This would improve clinical outcome, reduce medication-related errors and provide cost-effective treatment for the patients.

\section{Literature Review}

Bonkowski et al. (2013) conducted an observational study to measure the medi- 
cation administration error rate before and after the implementation of Bar-code medication administration (BCMA) in an emergency department (ED) as medication administration in the ED is complex and error-prone. Data collection is conducted through an observation protocol by the principal investigator with 3 blinded observers before and after implementation of BCMA. Documentation of the medication administration was compared with the physician order to ensure medication administration accuracy. The study revealed most medication errors were of minor severity [7]. The highest error rate came from antihistamine medications. Dosage errors were the most common error found in the study. The benefits of the implementation of BCMA in the ED have demonstrated significant reductions in medication errors, specifically wrong dose errors.

A pre and post-intervention study was conducted in the review done by Choo, Johnston \& Manias (2014) in two hospitals in Singapore to examine the effectiveness of an electronic medication record in reducing medication errors. Retrospective data of the two hospitals studied were analyzed. In one of the hospitals, the inpatient electronic medication record system was being implemented while the other hospital was using the paper-based medication record system. The study revealed there was no significant difference in the incidence of medication errors following the introduction of the electronic medication record system [8]. It suggested that further studies should be conducted to evaluate how this system can reduce medication error rates and improve medication safety.

A descriptive analytical study was conducted on 242 nurses to investigate the association of nurses' knowledge of Barcode Medication Administration (BCMA) and the implementation of BCMA in a hospital [9]. Self-designed questionnaires and checklists were used for data collection for measuring nurses' knowledge of BCMA and the rate of BCMA implementation in the hospital. The study revealed that there was a positive but weak correlation between nurses' knowledge of BCMA advantages and its implementation. It was found that the nurses' realization of the benefits of BCMA is insignificant and unacceptable. It also found that BCMA did not significantly reduce medication and prescription errors significantly which is not in correspondence to other research studies [9]. Educational courses should be instituted to improve the knowledge of nurses and better use of technology.

A case-control study was conducted by Gooder (2011) on 59 nurses to examine the perceived impact of barcoded medication administration system (BCMA) on nurses' ability to give medications, perceptions of medication errors, and nurses' satisfaction with BCMA process. Self-designed questionnaires were administered for data collection to the experimental group of nurses who use the BCMA system $(n=33)$ and the control group which BCMA has not been introduced. The study revealed that the overall satisfaction of the nurses following the implementation of the BCMA system has decreased. In addition, nurses faced decreased ability to visually see the medications due, as well as medications previously given following implementation of the BCMA. Although the use of BCMA systems is perceived as a promising technology, the findings have dif- 
fered from other studies which indicate that nurses are satisfied with the system [10]. Changes in workflow and work processes need to be re-engineered prior to the implementation and adoption of new technology.

Harrington, Clyne, Fuchs, Hardison \& Johnson (2013) evaluated Bar-Code Medication Administration (BCMA) implementation in a 260-bedded hospital on its progress to minimize medication errors to zero. The evaluation was carried out with the review of journals to create an evidence-based checklist with observational audits and interviews with nurses, followed by analyzing data and sharing of findings. There were three issues targeted for improvements and corrective actions were done and the results showed a compliance rate of $81 \%$ as compared to $72 \%$ that was recorded previously [11]. Through this project, the BCMA system implemented five years ago, using the evidence-based checklist from literature reviews, showed compliance with current evidence on its correct usage.

A literature review was conducted discussing the factors influencing medication administration safety, the influence of barcode technology and the successful implementation of barcode technology [12]. 11 articles were used, supported with interviews from subject matter experts. Barcode technology reduced dispensing errors up to $96 \%$ and documentation errors by $80.3 \%$. Barcode technology incorporated with electronic medication administration record has greatly improved the efficiency of medication administration and the safety aspect.

A summary of introducing electronic healthcare record (EHR) improved patient safety and implementing medication administration process using technology in a small rural England hospital [13]. The project was led by a Clinical Nurse Specialist (CNS) and core team members used literature reviews in nursing, pharmacy, and informatics. There were devices demonstrations by various vendors before procuring electronic medication administration record (eMAR) with a barcode scanner and training medication administration with automated medication dispensing machine and bar-coded medicine in a simulated patient room. The project was carried out in 3 phases which lasted for 36 months. There was a significant progressive successful medication scan rate of $93.95 \%$ in Phase $1 \%$ to $98 \%$ in Phase 3 [13]. The project was carried out in 36 months and included a process to pick up and make improvements to the errors observed. The project also demonstrated the importance of CNS' role in leading the team members and making the project a success.

Samaranayake, Cheung, Cheng, Lai, Chui \& Chueng (2014) led a mixed methods design study to assess the effects of a barcode-assisted medication administration (BCMA) system on the dispensing process and its users in a teaching hospital. Direct observation is used to assess the process of dispensing errors. Questionnaires and interviews were conducted to assess the attitudes of the nurses and pharmacist using the system. The findings revealed that additional steps were taken in the dispensing process and there were more errors following the implementing of the new system. In addition, nurses have perceived that the system is useful in improving the accuracy of medication administration. BCMA 
implementation might have slowed down and complicated the dispensing process. However, nurses believe that the system had improved the medication administration process [14]. It is recommended that ongoing evaluation of the changes should be in place to identify the weakness and staff should be educated on the benefits.

Based on the above reviews, various findings were indicative that with appropriate workflow, training, policy and procedures along with support and guidance, BCMA and e-MAR would greatly impact the medication administration process by significantly reducing medication errors.

\section{Rationale for BCMA and e-MAR}

Medication administration involves performing a series of complex steps for the medication to be administered. Due to the complexity of the process, there are high risks for errors to take place because of interruptions, the complexity of tasks, not following the five rights of medication administration and using workarounds. Workarounds implemented by nurses in the execution of required processes to achieve desired goals can lead to unintended outcomes, including errors [15]. Medication errors cause ADEs in patients with wrong medication administered and results in an escalation of cost in the health care system. In order to prevent ADEs and to reduce the rate of medication errors, many hospitals have started employing BCMA and e-MAR [5] [12]. To achieve error-free medication administration, the decision is made to employ BCMA and e-MAR technology to assist nurses, practitioners and pharmacists in the medication administration process that comprises of prescribing, transcribing, dispensing and administering of medications.

Traditionally, doctors prescribe medications by writing on the inpatient medication record (IMR). IMR will then be sent to the pharmacy for the pharmacist to prepare the medications and send to the ward. Nurses then prepare and administer the medications from the medication cart pushed into the patient's cubicle/room. This process seems to be less effective as there were many reported occurrences of medication errors as well as infection control issues especially for patients with undiagnosed transmission-based infections.

The primary function of e-MAR is to enable electronic prescription, administration and record of medications served to patients, thus replaces handwritten IMR that may be misread due to illegible handwriting and inappropriate use of abbreviations. Electronic MAR increases efficiency with the use of BCMA, while reducing medication errors caused by nurses who misread orders, miss, delay or even administer wrong medication and dosage. The development of the BCMA system is aimed to reduce medication administration errors, reduce the related costs incurred by the hospital and improve patient safety. Studies have shown that the rate of medication errors can be reduced up to $87 \%$ once an institute implements e-MAR [12]. Electronic MAR can also alert patient's drug allergies and generate audit reports and statistics such as medication inventory, missed or 
delayed dose of medication. Many hospitals reported the increased efficacy of barcode technology and improved medication safety with the successful implementation of BCMA [12] [16]. Hence, with these promising evidences, it creates sufficient standpoint for the innovation to be recommended. Table 1 illustrates the summary of advantages and disadvantages of BCMA and e-MAR system.

\section{Using BCMA and E-MAR for Medication Administration}

The recommended innovation is the implementation of BCMA and e-MAR

Table 1. Advantages and disadvantages of BCMA and e-MAR system.

\begin{tabular}{|c|c|}
\hline Pros & Cons \\
\hline Reduced errors in physician orders & Change process, resistance to change \\
\hline Reduced dosage errors & Non-compliance to change \\
\hline Reduced medication errors & Difficulty in using new technology \\
\hline Reduced prescription errors & Insufficient training and support \\
\hline Compliance resulted in satisfaction & Lack of knowledge \\
\hline $\begin{array}{l}\text { Improved the efficiency of medication } \\
\text { administration }\end{array}$ & $\begin{array}{l}\text { Unfriendly technology, inability to view pre- } \\
\text { viously served medication }\end{array}$ \\
\hline $\begin{array}{l}\text { Intelligent scanning devices scan the correct } \\
\text { dosage to be administered and alert if the wrong } \\
\text { dosage is dispensed }\end{array}$ & $\begin{array}{l}\text { Lack of appropriate policy and procedures and } \\
\text { workflow }\end{array}$ \\
\hline Improved patient safety & $\begin{array}{l}\text { BCMA implementation slow down and } \\
\text { complicated the dispensing process }\end{array}$ \\
\hline $\begin{array}{l}\text { Better outcome when incorporated with an } \\
\text { electronic medication administration system }\end{array}$ & $\begin{array}{l}\text { Use of equipment may hinder direct eye contact } \\
\text { with the patient }\end{array}$ \\
\hline Appropriate device and software required & Equipment malfunction \\
\hline Automated medication dispensing machine & System downtime \\
\hline Effective leadership for a better outcome & Cybersecurity issues \\
\hline Higher accuracy of medication administration. & Troubleshooting issues \\
\hline $\begin{array}{l}\text { Prevents wrong selection of patient as scanner } \\
\text { detects patient by scanning barcode identification } \\
\text { tag with medication }\end{array}$ & Lack of technical support \\
\hline $\begin{array}{l}\text { Prevents human errors caused by lack of } \\
\text { concentration and fatigue }\end{array}$ & Unfamiliar processes \\
\hline $\begin{array}{l}\text { Reduced cost related to adverse and sentinel } \\
\text { events }\end{array}$ & $\begin{array}{l}\text { Lack of communication and understanding by } \\
\text { employees }\end{array}$ \\
\hline Enhance Infection control practices & $\begin{array}{l}\text { No integration between information technology } \\
\text { and nursing processes }\end{array}$ \\
\hline $\begin{array}{l}\text { Enhance compliance with training, policy and } \\
\text { procedure in place }\end{array}$ & Change process, resistance to change \\
\hline $\begin{array}{l}\text { Alert patient's drug allergies, generate audit } \\
\text { reports and statistics such as medication } \\
\text { inventory, missed or delayed dose of medication }\end{array}$ & Non-compliance to change \\
\hline
\end{tabular}


system with the use of a Bluetooth barcode scanner and a hand-held device such as IPAD/tablet. The purpose of e-MAR is to prevent errors caused by physicians' illegible handwriting, missed/ delayed dose of medication. It also ensures the accurate dose is being served to patients with available information about the medication and for correct documentation. The barcode scanner prevents the wrong selection of the patient and ensures that the correct medication and dose is being served and prevents human errors caused by lack of concentration [11]. The handheld device replaces the hard copy inpatient medication record and the bulky computer on wheels with an attached scanner that is cumbersome and hinders eye contact with the patient while checking the two identifiers (Identification number and name). This innovation is aimed to reduce medication error significantly and increases the staff compliance in ensuring that correct medication is served to the right patients adhering to the five rights of medication administration [17] [18]. The implementation of the innovation consists of the product introduction and process modifications.

\subsection{Selection of Hardware and Software}

The selection of the product includes the hardware and the software. The product identification and evaluation require both the Information Technologist (IT) and Registered Nurses (RN) respectively. The IT is needed to identify the handheld device (IPAD/Tablet) which has a suitable specification to install the software. Subsequently, the RNs who are the end users have an obligation to evaluate two different types of Bluetooth barcode scanners and handheld devices to compare the speed, ease of use and select the most appropriate device for the implementation of BCMA and e-MAR. The other aspect of product evaluation includes the selection of software program that is easily installed, maintained, encrypted and able to form communication links between physicians, pharmacists and nurses to ensure a closed loop system [13]. Once this is established, the medications are labelled with barcodes by pharmacists.

\subsection{Education and Training}

Education and training include a structured program to teach the physicians, pharmacists and nurses [13] [19]. This is essential to ensure that the entire system is safe for use and knowledge of system troubleshooting would reduce process failure in the event of equipment failure.

\subsection{Process Modification}

The process modification including a change in the way medication is served. The current medication process involves prescription in inpatient medication record (IMR) by a physician, routing prescriptions and transcribing the prescription at the pharmacy dispensed by pharmacists, administration of medicines and monitoring of patients by nurses [20] [21]. With the process modifica- 
tion, the physician will order the medication at the e-MAR in the physician's module, and the medication will be automatically routed to the pharmacist's module.

Once the pharmacist receives the order and clicks on the patient's details and specific orders, the medication labels will be printed. The advantage of the system is that the medication doses can be entered in the form that it is available. For example, if the physician has ordered $1 \mathrm{~g}$ paracetamol, the pharmacists can input the available dose $500 \mathrm{mg}$ per tablet and ensure that two tablets are retrieved for the proper administration of order by pre-setting in the software. This is achieved by the system prompting the nurses to scan the labelled medication for the second time to attain the desired dosage as per prescription. The medication indications, side effects and other pertinent information are also readily available for nurses to refer when the medication order is clicked in the ward.

In the ward, during the medication administration time, the medication that is due to be served and patients with drug allergy will be highlighted with colour (e.g. yellow, red, etc.) to serve as a reminder. The first step towards safe medication administration begins with the scanning of the patient's wrist tag. Once the wrist tag is scanned, the patient's information and medication record will automatically launch. The two identifiers verification may be done with the hand-held device (IPAD/Tablet) that shows patients records. Pre-medication assessment for pain, blood pressure, and so forth may be performed at this stage. Once the verifications and evaluations are done, the nurse may proceed to retrieve the medications for the patient. The medication label is scanned, and doses are scanned accordingly as per order. After that, the preparation is verified by a second registered nurse who is required to enter her personal pin code to confirm that the medication is checked by her/him before serving the patient. At this stage, the nurse may refer to the handheld device (IPAD/Tablet) for indication and other pertinent information about the medication, and educate the patient accordingly. Patient education is vital in the identification of adverse drug reactions (ADR) and any other unforeseen events that may arise. Modification of the process and the inclusion of additional steps safeguard the patients and the healthcare professionals from any mishaps.

\subsection{Infection Control}

The use of non-touch technique verification with handheld devices (IPAD/Tablet) assures a good infection control practice. The new practice eliminates the cumbersome disinfection of mobile cart entirely. The medication cart will be stationed outside the patients' cubicle to prevent contact with unknown airborne infectious diseases and cross-contamination to other patients [22]. The hand-held devices (IPAD/ Tablet) and Bluetooth barcode scanner are conveniently disinfected with alcohol wipes because of the size of the device.

\subsection{Risk of Malfunction}

A study conducted by Rack, Dudjak and Wolf (2012) revealed that more than 
half of the nurses had administered medications without scanning either the medication or the patient during their shift. The reasons for such negligence include technology failures such as the inability to scan labels due to malfunctioning software, damaged labels, and patient's isolation precaution status (scanner with the computer on wheels used) [23]. Hence, the inefficiency of the technology may impair the use, and reduce the expected safety benefits. The malfunction may result in termination or delay in the clinical operation or even requiring the user to opt for manual medication administration during downtime [23]. These problems can be precluded with proper preventative maintenance and through a proficient reporting system that alerts any technical issues in advance in order to fix the malfunction promptly.

\subsection{Cybercrime}

As indicated earlier, the barcode medication administration cannot be used without e-MAR. When the patient's information is held online, it is prone to cyber-attacks or ransomware. Hackers sometimes hold medical data hostage until they receive the amount of money, their demands resulting in the slowing down of work, unduly stress and confidentiality issues [24]. When implementing the new innovative device, it will be necessary to ensure that a software security protocol is implemented to prevent cyber-crimes. In this case, there will be a need for professional IT personnel to undertake security measures for the patient's medical records and confidential data.

\subsection{High Cost}

BCMA and e-MAR is an exclusive innovation that incurs an excessive cost to many organizations. Adequate financial strategies need to be established to acquire the required technical support, equipment and software to support the innovation. The public sector may request for government funding in consideration of additional developments [25]. However, this may be unfeasible for the private sector. As such, this may delay the launch of costly technological advancement.

\subsection{Staff Resistance}

Staff resistance to the implementation of BCMA and e-MAR is a significant barrier that is experienced by many health care facilities. Resistance can be caused by poor communication. The staff feel overwhelmed due to the change of roles, sometimes because of the employees' negative perception about the technology [26] [27]. To overcome this barrier, the management will have to assure that proper change management and communication plan is employed to reassure the healthcare professionals involved.

\subsection{Training}

Training of "super users" who may provide technical assistance in the absence of 
IT will facilitate the implementation of BCMA and e-MAR. It is vital to conduct adequate training for all health care professionals to build their confidence. Proactive vendors and project team are essential to assure that specific modification is addressed in time when necessary [12] [28]. This would boost the morale of healthcare professional and encourage them to venture into the innovation.

\subsection{Mechanical Issues}

There have been complaints that barcodes on the medication are placed on surfaces that are difficult to be scanned [26] [29]. The investment in a good label printer that prints barcodes accurately and the training of pharmacy technicians to affix medication labels on a suitable surface would reduce unduly medications returned to the pharmacy.

\section{Cultivating a Process Change}

Change is the effect of innovation that emerges in the launch of an invention. The success of innovation involves effective leadership, change management and support of staffs to embrace the change. According to Bucciarelli (2015), Kotter's eight-stage model is the most popular change model embraced by many organizations as this model begins with the reflection of failure in change and creates a positive attribute to transform the error into stages that create successful change. Kotter's (2012) eight stages of leading change include establishing a sense of urgency, creating the guiding coalition, developing a vision and strategy, communicating the change vision, empowering broad-based action, generating short-term wins, consolidating gains and producing more change and anchoring innovative approaches in the culture.

The implementation of BCMA and e-MAR in the organization requires cooperation from the management and staff. Hence, to accomplish the goals and embrace transformation, it is crucial for the management to understand the crisis in the increasing number of medication errors. This would prevent complacency in the organization. It is evident that observable catastrophes immensely assist in acquiring attention and escalating the urgency levels. Thus, statistical presentation of the number and types of medication errors, adverse events and seriously reportable events (SRE) would substantiate the significance in the implementation of BCMA and e-MAR. Guiding coalition includes authority, expertise, reliability and strong leadership. This enhances the leader's proficiency in being a change agent and working with the rest of the team members for a successful change [29]. Hence, project development requires the formation of a team with members from various departments and management levels with designated roles and responsibilities that would create a model to embrace change. Development of vision and strategy can only be achieved when there is a clear understanding of the needs and reasons for change [30] [31]. Subsequently, an appropriate communication strategy should be derived to share the information throughout the organization for staff and management to buy in the idea. 
The BCMA and e-MAR development comprises of the sharing of the medication error statistics, creating a mutual consent among the staff and management, and generating a performance indicator that aims to reduce medication errors. Qualitative and quantitative details of the products (Scanning device and handheld device) and the end users (registered nurses, physicians and pharmacists) should be shared to prepare the staffs to accept the change in the process. The timeline to launch BMCA and e-MAR into the system and the cost incurred regarding the purchase of hardware and software, process changes and staff training should be budgeted. Lastly, customers' and staff's satisfaction rate should be evaluated. This aspect of the clinical innovation is critical, as the goal to reduce medication error is set with the aim to provide quality care for the patients. Therefore, it is necessary to collect relevant data to evaluate the success of the BCMA and e-MAR once it is launched.

Communication is the key factor that creates the path to the success or failure of any initiatives. Henceforth, a well-structured communication design that elucidates the benefits of the change and active listening of the staff and management feedback, and staff empowerment is fundamental [32]. It is crucial to identify obstacles and eliminate them so that staff will not be re-routed from the desired outcome. In the BCMA and e-MAR implementation, constant technical and moral support is required to motivate the staffs to adapt to the change.

Identifying supportive staff and team members coupled with appropriate rewards and recognition would encourage them to assist in the project implementation process [26]. This will demonstrate the impact, importance and assist in alleviating the effect of staff and team members who are unsupportive. Once the BCMA and e-MAR are implemented, it would be beneficial to consolidate all the feedback and suggestions on the pros and cons of the system to further improvise the system [33] [34]. The system performance may be monitored and analyzed with the amount of usage and discussions with the staff.

Institutionalized innovative approaches, with strong leadership and identification of the leader's successor, ensure continuity in the transformation process [32]. Embracing an organizational change begins with the alteration of norms and values followed by the organization culture. Once the organizational culture has been articulated with corporate success, the change effort becomes more achievable. Thus, the implementation of BCMA and e-MAR requires the adoption of a strong organizational culture that embraces innovation. Organizational change is a very fluid process affecting numerous constituents to be strategically manoeuvred [34]. It is common for failures to occur when organizations try to innovate and attempt a change. However, it is important to note that change is constant and should be supported to persevere with the emerging healthcare advancements.

\section{An evaluation Framework for BCMA and e-MAR Practices}

As mentioned by Nelson-Brantley and Ford (2016), change is a social, dynamic 
and interactive process. As such, the concept of leading change may be evaluated by exploring specific and collective information at the micro, meso and macro level of a system. The evaluation framework provides an evidence-based results for assessing the innovation project design. It will enable the reallocation of resources and improve the project plan as needed.

Project evaluation is a systematic process intended to examine the effectiveness, efficiency and appropriateness of the clinical innovation that is meant to improve clinical outcomes [35]. The evaluation also enables the identification of strategies to improve the engagement of healthcare professionals in the implementation of the BCMA and e-MAR system. The approaches of gathering information may be collected via formative, summative process evaluation. The evaluation design includes the background of project, goals and objectives, the purpose of evaluation, parameters of evaluation, project outcome, evaluation questions, evaluation criteria, communication of findings and evaluation timeframe [36]. The evaluation criteria determine the quality, impact and outcome of the clinical innovation [37]. These measures are required to comprehend the success and failure of the project.

\section{Determining the Degree of Success of BCMA and e-MAR}

Based on the evaluation design, assessment criteria, feedback and audits, the results of findings may be tabulated to determine the degree of success of the innovation and the project plan [31]. Appraisal of findings through various methods of data collection such as product and process utilization, software loading and lagging time, awareness of healthcare professionals, change in attitudes, knowledge, satisfaction rate of healthcare professionals and patients, sustainability of innovation and reduced costs in patient care outcome related to reduce medication error enables measurement of the project a success. Qualitative and quantitative analysis of these statistical data provides evidence-based information about the measurement of success rate.

\section{Discussion}

Upon completion of the project upholding the steps discussed above, a pilot study was initiated in one unit consisting of 43 nursing staff and multidisciplinary doctors within an anonymous organization. Prior to the pilot run, a roadshow was conducted for staffs to evaluate the product and process of the new medication management system. The pilot study was conducted for a period of two months in one unit. To ensure the effectiveness of the BCMA and e-MAR system, a project team was deployed to the unit to address any concerns raised by the nurses and doctors. On-the-job training, close supervision, clinical guidance as well as nursing informatics personnel were readily available to troubleshoot and resolve issues raised. Constant feedbacks were gathered from end-users to evaluate the effectiveness of the new system as well as the process.

All medication prescribed was verified by the pharmacists' team before the 
medications could be served. The system provided indications and contraindications for each medication, its side effects and adverse reactions as well as correct dilution and diluents for intravenous therapy. The success of the innovation was measured with medication administration audits, staff feedback and the number of hospital occurrence reports raised with regards to medication errors. The BCMA and e-MAR system was widely accepted by the staff as the number of errors reported was significantly reduced. The staff mentioned that the continuous support from the project team increased their confidence to use the new system. There was only 1 near missed error reported over the two months trial period. The investigation revealed that there was non-compliance observed due to understaffing and fatigue.

In the initial stage, resistance was seen among physicians as the added step of prescribing in the system was time-consuming. Other contrary feedback included that the system was slow to log in, load and toggle as the Wi-Fi connection was rather slow. It prolonged medication serving time as well as caused dissatisfaction among patients who received their routine medication later than usual. Although, infection control matters were addressed by the use of handheld devices such as iPad and scanner that was conveniently cleaned before its next use. The nurses expressed the difficulty in transporting the devices and medication cups/trays from room to room to serve medication. They verbalized that a carrier to hold the devices would assist them away from fumbling with multiple items. Overall feedback was gathered and $88.3 \%$ of staff were in favor of the BCMA and e-MAR system and the remaining $11.7 \%$ were against the idea due to the inconvenience discussed above. Further studies in numerous departments with bigger sample size and extensive study period are recommended to identify other issues faced by staff and also to resolve the internet connection lag before the implementation of BCMA and e-MAR system.

\section{Conclusions}

Medication safety is the responsibility of all members of the healthcare team. It is evident from several studies that medication error is one of the leading causes of death in hospitalized patients. The BCMA and e-MAR system would effectively reduce the number of errors with efficient technology. With appropriate training and support, the healthcare team would be able to adhere to a high standard through the use of technological advancement in healthcare and reduce the number of reported errors.

Although in the beginning stage, the implementation process of the BCMA and e-MAR may be cumbersome and cause delays in medication administration, it is doable once it is recognized as being part of the routine care standards. Above all, the success and failure of this clinical innovation depend greatly on the communication strategies and the evaluation design that measures the need of improvisation of the process. The expected outcome of the innovation is to assist the healthcare professionals to perform their clinical roles efficiently and 
provide cost-effective treatment outcome to the patients.

\section{Conflicts of Interest}

The authors declare no conflicts of interest regarding the publication of this paper.

\section{References}

[1] Center for Drug Evaluation and Research (2016) Medication Error Reports. https://www.fda.gov/Drugs/DrugSafety/MedicationErrors/ucm080629.htm

[2] ISMP Guidelines (n.d.) https://www.ismp.org/Tools/guidelines/

[3] Anderson, P. (2010) Medication Errors: Don't Let Them Happen to You. Journal of American Nurses Association, 5, 17-21.

https://www.americannursetoday.com/medication-errors-dont-let-them-happen-to -you/

[4] Hassink, J.J., Jansen, M.M. and Helmons, P.J. (2012) Effects of Barcode-Assisted Medication Administration (BCMA) on Frequency, Type and Severity of Medication Administration Errors: A Review of the Literature. European Journal of Hospital Pharmacy, 19, 489-494. https://doi.org/10.1136/ejhpharm-2012-000058

[5] Gorgich, E.A., Barfroshan, S., Ghoreishi, G. and Yaghoobi, M. (2015) Investigating the Causes of Medication Errors and Strategies to Prevention of Them from Nurses and Nursing Student Viewpoint. Global Journal of Health Science, 8, 220. https://doi.org/10.5539/gjhs.v8n8p220

[6] Kale, A., Keohane, C.A., Maviglia, S., Gandhi, T.K. and Poon, E.G. (2012) Adverse Drug Events Caused by Serious Medication Administration Errors. BMJ Quality \& Safety, 21, 933-938. https://doi.org/10.1136/bmjqs-2012-000946

[7] Bonkowski, J., Carnes, C., Melucci, J., Mirtallo, J., Prier, B., Reichert, E., et al. (2013) Effect of Barcode-Assisted Medication Administration on Emergency Department Medication Errors. Academic Emergency Medicine, 20, 801-806.

https://doi.org/10.1111/acem.12189

[8] Choo, J., Johnston, L. and Manias, E. (2014) Effectiveness of an Electronic Inpatient Medication Record in Reducing Medication Errors in Singapore. Nursing \& Health Sciences, 16, 245-254. https://doi.org/10.1111/nhs.12078

[9] Ehteshami, A., Azizi, M., Amini, M., Fazeli, T. and Kasaei, M. (2014) Barcode Medication Administration and Patient Safety: A Case Study in Iran. International Journal of Health System and Disaster Management, 2, 186-191.

https://doi.org/10.4103/2347-9019.142208

[10] Gooder, V. (2011) Nurses' Perceptions of a (BCMA) Bar-Coded Medication Administration System: A Case-Control Study. Online Journal of Nursing Informatics $(O J N I), 15,125-137$.

[11] Harrington, L., Clyne, K., Fuchs, M., Hardison, V. and Johnson, C. (2013) Evaluation of the Use of Bar-Code Medication Administration in Nursing Practice Using an Evidence-Based Checklist. The Journal of Nursing Administration, 43, 611-617.

[12] Leung, A., Denham, C., Gandhi, T., Bane, A., Churchill, W., Bates, D. and Poon, E. (2015) A Safe Practice Standard for Barcode Technology. Journal of Patient Safety, 11, 89-99.

[13] Richardson, B., Bromirski, B. and Hayden, A. (2012) Implementing a Safe and Re- 
liable Process for Medication Administration. Clinical Nurse Specialist, 26, 169-176. https://doi.org/10.1097/NUR.0b013e3182503fbe

[14] Samaranayake, N., Cheung, S., Cheng, K., Lai, K., Chui, W. and Cheung, B. (2014) Implementing a Bar-Code Assisted Medication Administration System: Effects on the Dispensing Process and User Perceptions. International Journal of Medical Informatics, 83, 450-458. https://doi.org/10.1016/j.ijmedinf.2014.03.001

[15] Debono, D.S., Greenfield, D., Travaglia, J.F., Long, J.C., Black, D., Johnson, J. and Braithwaite, J. (2013) Nurses' Workarounds in Acute Healthcare Settings: A Scoping Review. BMC Health Services Research, 13, 175. https://doi.org/10.1186/1472-6963-13-175

[16] Rack, L., Dudjak, L. and Wolf, G. (2012) Study of Nurse Workarounds in a Hospital Using Bar Code Medication Administration System. Journal of Nursing Care Quality, 27, 232-239. https://doi.org/10.1097/NCQ.0b013e318240a854

[17] Seibert, H., Maddox, R., Flynn, E. and Williams, C. (2014) Effect of Barcode Technology with Electronic Medication Administration Record on Medication Accuracy Rates. American Journal of Health-System Pharmacy, 71, 209-218. https://doi.org/10.2146/ajhp130332

[18] Ministry of Health Singapore (2006) https://www.moh.gov.sg/docs/librariesprovider4/guidelines/medication-safety.pdf

[19] Buerhaus, P.I. (2013) Human Factors Engineering, Bar Coding Medication Administration, and Nursing: An Interview with Drs. Richard Holden and Laurie L. Novak. Nursing Economics, 31, 190-193.

http://go.galegroup.com.libraryproxy.griffith.edu.au/ps/i.do?p=EAIM\&sw=w\&u=gr ifith\&v=2.1\&it=r\&id=GALE\%7CA341687285\&sid=summon\&asid=dae5e48fb7d37b aac592db4e4474aa77

[20] Nanji, K.C., et al. (2009) Overcoming Barriers to the Implementation of a Pharmacy Bar Code Scanning System for Medication Dispensing: A Case Study. Journal of the American Medical Informatics Association, 16, 645-650. https://doi.org/10.1197/jamia.M3107

[21] Hunter, K. (2011) Implementation of an Electronic Medication Administration Record and Bedside Verification System. Online Journal of Nursing Informatics (OJNI), 15. http://ojni.org/issues/?p=672

[22] Gribogiannis, M. (2011) Infection Prevention Issues Associated with Point of Care Barcode Scanner Mobile Computers. American Journal of Infection Control, 39, E148-E149. https://doi.org/10.1016/j.ajic.2011.04.252

[23] Tseng, K.J., et al. (2012) Implementation and Evaluation the Effectiveness of the Bar-Coded Medication Administration System in a Medical Center. NI 2012: Proceedings of the 11 th International Congress on Nursing Informatics, 2012, 416.

[24] Ducker, M., Sanchez, C. and Taylor, S.R. (2013) Pros and Cons of E-Prescribing in Community Pharmacies. US Pharmacist, 8, 4-7.

[25] Sakowski, J.A. and Ketchel, A. (2013) The Cost of Implementing Inpatient Bar Code Medication Administration. The American Journal of Managed Care, 19, 38-45. http://www.ajmc.com/journals/issue/2013/2013-1-vol19-n2/the-cost-of-implemeni ng-inpatient-bar-code-medication-administration/P-3\#sthash.9uWOV8SA.dpuf

[26] Ventura, M.L. (2011) The Electronic Medical Record: Pros and Cons. The Journal of Maternal-Fetal \& Neonatal Medicine, 24, 163-166. https://doi.org/10.3109/14767058.2011.607582

[27] VanderKooi, M.M. (2014) An Evidence-Based Evaluation of Medication Barcode 
Scanning Acceptance in a Community Hospital. Doctoral Dissertations, Grand Valley State University, Allendale, Michigan.

[28] Shah, K., Lo, C., Babich, M., Tsao, N.W. and Bansback, N.J. (2016) Bar Code Medication Administration Technology: A Systematic Review of Impact on Patient Safety When Used with Computerized Prescriber Order Entry and Automated Dispensing Devices. The Canadian Journal of Hospital Pharmacy, 69, 394-402. https://doi.org/10.4212/cjhp.v69i5.1594

[29] Dallas, H.J. (2015) Mastering the Challenges of Leading Change: Inspire the People and Succeed Where Others Fail. Wiley, Hoboken, NJ. https://doi.org/10.1002/9781119222040

[30] Kotter, J.P. (2012) Leading Change. Harvard Business Review Press, Boston, MA.

[31] Webster, M. (2012) Successful Change Management-Kotter's 8-Step Change Model. https://www.leadershipthoughts.com/kotters-8-step-change-model/

[32] Bucciarelli, L. (2015) A Review of Innovation and Change Management: Stage Model and Power Influences. Universal Journal of Management, 3, 36-42. https://doi.org/10.13189/ujm.2015.030106

[33] Nelson-Brantley, H.V. and Ford, D.J. (2016) Leading Change: A Concept Analysis. Journal of Advanced Nursing, 73, 834-846. https://doi.org/10.1111/jan.13223

[34] Stanleigh, M. (2013) Leading Change. The Journal for Quality and Participation, 36, 39-40.

[35] Agency for Clinical Innovation (2013) Understanding Program Evaluation: An ACI Framework.

https://www.aci.health.nsw.gov.au/_data/assets/pdf_file/0008/192437/FrameworkProgram-Evaluation.pdf

[36] Cheah, S. (2016) Framework for Measuring Research and Innovation Impact. Innovation, 18, 212-232. https://doi.org/10.1080/14479338.2016.1219230

[37] Health Quality Ontario (2016) Evaluation of Innovative Practices: Process and Methods Guide in Support of Advancing a Health Links Approach. http://www.hqontario.ca/Portals/0/documents/qi/health-links/evaluation-innovativ e-practices-process-guide-en.pdf 